\begin{tabular}{|c|l|}
\hline Title & Spontaneous edge current in a small chiral superconductor with a rough surface \\
\hline Author(s) & Suzuki, Shu-Ichiro; A sano, Y asuhiro \\
\hline Citation & $\begin{array}{l}\text { Physical Review B, 94(15), 155302 } \\
\text { https://doi.org/10.1103/PhysRevB.94.155302 }\end{array}$ \\
\hline Issue Date & 2016-10-07 \\
\hline Doc URL & http://hdl.handle.net/2115/63644 \\
\hline Rights & @2016 A merican Physical Society \\
\hline Type & article \\
\hline File Information & PhysRevB.94.155302.pdf \\
\hline
\end{tabular}

Instructions for use 


\title{
Spontaneous edge current in a small chiral superconductor with a rough surface
}

\author{
Shu-Ichiro Suzuki ${ }^{1}$ and Yasuhiro Asano ${ }^{1,2,3}$ \\ ${ }^{1}$ Department of Applied Physics, Hokkaido University, Sapporo 060-8628, Japan \\ ${ }^{2}$ Center for Topological Science and Technology, Hokkaido University, Sapporo 060-8628, Japan \\ ${ }^{3}$ Moscow Institute of Physics and Technology, 141700 Dolgoprudny, Russia \\ (Received 17 February 2016; revised manuscript received 31 May 2016; published 7 October 2016)
}

\begin{abstract}
We study theoretically the spontaneous edge current in a small chiral superconductor with surface roughness. We obtained self-consistent solutions of the pair potential and the vector potential by solving the quasiclassical Eilenberger equation and the Maxwell equation simultaneously. We then employed them to calculate numerically the spatial distribution of the chiral edge current in a small superconductor. The characteristic behavior of the spontaneous edge current depends strongly on the symmetries of the order parameters such as chiral $p$-, chiral $d$-, and chiral $f$-wave pairing. The edge current is robust under the surface roughness in the chiral $p$ - and chiral $d$-wave superconductors. In the chiral $d$-wave case, the surface roughness tends to flip the direction of the chiral current. On the other hand, the edge current in a chiral $f$-wave superconductor is fragile when there is surface roughness. We also discuss the temperature dependence of a spontaneous magnetization, which is a measurable value in standard experiments.
\end{abstract}

DOI: 10.1103/PhysRevB.94.155302

\section{INTRODUCTION}

The experimental detection of a spontaneous edge current could be direct evidence of chiral superconductivity. A number of Cooper pairs sharing a specific angular momentum carry the spontaneous edge current in chiral superconductors $[1,2]$, which can be experimentally measured as spontaneous magnetization. Strontium ruthenate $\mathrm{Sr}_{2} \mathrm{RuO}_{4}$ is a leading candidate for a chiral $p$-wave superconductor [3-5] whose pair potential is described by $\Delta\left(k_{x} \pm i k_{y}\right)=\Delta e^{i \chi \theta}$ in momentum space. Here $k_{x}=\cos \theta\left(k_{y}=\sin \theta\right)$ is the normalized wave number in the $x(y)$ direction, and $\Delta$ is the amplitude of the pair potential. The topological Chern number $\chi=1$ or -1 corresponds to the angular momentum of a Cooper pair. In addition to chiral $p$-wave superconductivity, the possibilities of chiral $d$-wave $(\chi= \pm 2)$ and chiral $f$-wave $(\chi= \pm 3)$ superconductivity have been discussed in recent experiments [6-16]. Several theories have suggested that the amount of edge current becomes smaller in a chiral superconductor with a larger $|\chi|[17,18]$. However, unfortunately, no spontaneous chiral current has yet been experimentally observed $[19,20]$.

The absence of spontaneous magnetization in experiments has mainly been attributed to three effects: (i) the Meissner screening of the edge current by the bulk superconducting condensate, (ii) the reduction of the chiral current by the potential disorder near the surface of a superconductor, and (iii) the complicated electronic structures of superconductors. The first effect was partially studied by Matsumoto and Sigrist [1]. They theoretically confirmed a reduction in the edge current caused by the Meissner effect in a chiral $p$-wave superconductor. However, the resulting spontaneous magnetization is large enough to be measured in experiments. The second effect is linked to the issue of the intrinsic angular momentum in the ${ }^{3} \mathrm{He}-\mathrm{A}$ phase $[21,22]$. Experimentally it is difficult to make a superconducting sample with a specular surface. For instance, a small ruthenate superconductor cluster can be fabricated by using the focused ion beam technique $[23,24]$, which would seriously damage the sample quality near the surface. Several theoretical papers have already suggested the presence of edge states in a chiral $p$-wave superconductor when there is surface roughness $[25,26]$. On the other hand, when a chiral $p$-wave superconductor is covered by a clean normal metal, the chiral current is dramatically reduced [27]. The third effect has been discussed specifically in $\mathrm{Sr}_{2} \mathrm{RuO}_{4}$. It has been known that the gap anisotropy $[28,29]$ and the multiband structures [30] suppress the chiral edge current. Even today, we do not know how the Meissner screening and the surface roughness reduce the edge current in chiral $d$ - and $f$-wave superconductors. In previous papers [31,32], we studied the Andreev bound states [33-38] (ABSs) in time-reversal nonchiral superconductors characterized by $d_{x^{2}-y^{2}}$-wave or $p_{x}$-wave pair potentials. We found that the ABSs in a $p_{x}$-wave superconductor are robust even in the presence of surface roughness, whereas those in a $d$-wave superconductor are fragile against surface roughness. This conclusion is well explained by the symmetry of the Cooper pairs induced near the surface. However, it is unclear if it is possible to generalize our conclusions straightforwardly to chiral superconductors. We will address these issues in the present paper.

In this paper, we theoretically study the spontaneous edge currents and the spontaneous magnetization in a small chiral superconducting disk based on the quasiclassical Eilenberger formalism. To discuss the relation between the pairing symmetry and the sensitivity of the chiral edge current to the surface roughness, we consider the simple chiral order parameters on a circular shaped Fermi surface. By solving the Eilenberger and Maxwell equations self-consistently and simultaneously, we obtain the spatial profiles of the chiral edge currents and the temperature dependence of a spontaneous magnetization. The surface roughness is considered in terms of the impurity self-energy of a quasiparticle. To define the magnetization of a sample, we need to consider a finite-size superconductor such as disks. Moreover, setting the radius of a disk to be comparable to the coherence length allows us to justify the assumption that there is no chiral-domain wall in a disk. We conclude that the robustness of the spontaneous edge current depends strongly on the paring symmetry. In a chiral $p$-wave superconductor, the amplitude of the chiral current in a disk with a rough surface is comparable to that in a disk with a specular surface. In a 
chiral $d$-wave superconductor, there are two edge channels in a disk with a specular surface. They carry the chiral currents in opposite directions. In the presence of surface roughness, one channel near the surface disappears and the other channel far from the surface carries the robust chiral current. We show that the surface roughness changes the net-current direction in a chiral $d$-wave disk. The edge current in a chiral $f$-wave superconductor is fragile in the presence of surface roughness. The effects of Meissner screening on the chiral edge current depend on the spatial current distribution near the surface. When the current decreases monotonically with increases in the distance from the surface, the Meissner effect always reduces the chiral current. On the other hand, when the chiral current changes its direction as a function of distance from the surface, the Meissner screening effect becomes weaker. Such a complicated current distribution causes the self-screening effect among edge currents flowing in opposite directions.

This paper is organized as follows. In Sec. II, we explain the quasiclassical Eilenberger formalism and define the spontaneous magnetization of a small superconducting disk. In Sec. III, we present results obtained using non-selfconsistent simulations (i.e., with a homogeneous pair potential and without a vector potential). In Sec. IV, we discuss the spontaneous edge current in a superconducting disk with a specular surface. In Sec. V, we study the effects of surface roughness on the spontaneous edge current. In Sec. VI, we demonstrate the temperature dependence of a spontaneous magnetization, which is a measurable value in experiments. In Sec. VII, we summarize this paper.

\section{QUASICLASSICAL EILENBERGER THEORY}

Let us consider a small chiral superconducting disk as shown in Fig. 1. We assume that there are no chiral domains by choosing the radius of the disk $R$ to be comparable to the coherence length $\xi_{0}=\hbar v_{F} / 2 \pi T_{c}$, where $v_{F}$ is the Fermi velocity and $T_{c}$ is the superconducting transition temperature. We apply the quasiclassical Green's function theory of superconductivity [39] to calculate the edge current of a chiral superconductor. In an equilibrium superconductor, the Eilenberger equation takes the form

$$
i v_{F} \boldsymbol{k} \cdot \nabla_{\boldsymbol{r}} \check{g}+[\check{H}+\check{\Sigma}, \check{g}]_{-}=0,
$$

where $v_{F}$ is the Fermi velocity, $\boldsymbol{k}$ is the unit wave vector on the Fermi surface, and $[\alpha, \beta]_{-}=\alpha \beta-\beta \alpha$. We employ the isotropic cylindrical Fermi surface (i.e., no $k_{z}$ dependence) as studied in Ref. [1] because most chiral superconductors are layered materials. Throughout this paper, we use the set of

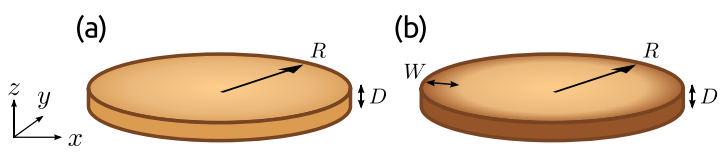

FIG. 1. Schematics of superconducting disks. The disk with a specular surface and that with a rough surface are shown in panels (a) and (b), respectively. The radius and thickness of a disk are denoted by $R$ and $D$, respectively. The width of the disordered region is denoted by $W$ in panel (b). The radius is small enough to allow us to assume that there is no chiral domain in the disk. units $\hbar=k_{B}=c=1$, where $2 \pi \hbar$ is the Planck constant, $k_{B}$ is the Boltzmann constant, and $c$ is the speed of light. The matrices $\check{g}$ and $\check{H}$ are defined as follows:

$$
\begin{gathered}
\check{g}\left(\boldsymbol{r}, \boldsymbol{k}, i \omega_{n}\right)=\left[\begin{array}{cc}
\hat{g}\left(\boldsymbol{r}, \boldsymbol{k}, i \omega_{n}\right) & \hat{f}\left(\boldsymbol{r}, \boldsymbol{k}, i \omega_{n}\right) \\
-\hat{f}\left(\boldsymbol{r}, \boldsymbol{k}, i \omega_{n}\right) & -\underset{\sim}{\hat{g}}\left(\boldsymbol{r}, \boldsymbol{k}, i \omega_{n}\right)
\end{array}\right], \\
\check{\sim}\left(\boldsymbol{r}, \boldsymbol{k}, i \omega_{n}\right)=\left[\begin{array}{cc}
\hat{\xi}\left(\boldsymbol{r}, \boldsymbol{k}, i \omega_{n}\right) & \hat{\Delta}(\boldsymbol{r}, \boldsymbol{k}) \\
\underset{\sim}{\hat{\Delta}}(\boldsymbol{r}, \boldsymbol{k}) & \underset{\sim}{\hat{\xi}}\left(\boldsymbol{r}, \boldsymbol{k}, i \omega_{n}\right)
\end{array}\right],
\end{gathered}
$$

with $\hat{\xi}\left(\boldsymbol{r}, \boldsymbol{k}, i \omega_{n}\right)=\left[i \omega_{n}+e v_{F} \boldsymbol{k} \cdot \boldsymbol{A}(\boldsymbol{r})\right] \hat{\sigma}_{0}$, where $\omega_{n}=(2 n+$ 1) $\pi T$ is the Matsubara frequencies with $n$ being an integer, $T$ is the temperature, $\hat{\Delta}$ represents the pair potential, $\hat{\sigma}_{0}$ is the $2 \times 2$ identity matrix in spin space, and $\boldsymbol{A}$ is the vector potential induced by the chiral edge current. We introduce the

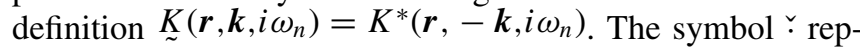
resents a $4 \times 4$ matrix structure in particle-hole space and the symbol $\hat{r}$ represents a $2 \times 2$ matrix structure in spin space.

We consider three chiral superconductors with different pairing symmetries: spin-triplet chiral $p$-wave, spin-singlet chiral $d$-wave, and spin-triplet chiral $f$-wave pairings. In the spin-triplet superconductor, we assume that the pairing interactions work between two electrons with opposite spins. This assumption does not loose any generality of the argument below. The pair potential are described by

$$
\hat{\Delta}(\boldsymbol{r}, \theta)= \begin{cases}\Delta(\boldsymbol{r}, \theta) \hat{\sigma}_{1} & \text { for a spin triplet, } \\ \Delta(\boldsymbol{r}, \theta) i \hat{\sigma}_{2} & \text { for a spin singlet, }\end{cases}
$$

where $\hat{\sigma}_{j}$ for $j=1-3$ are the Pauli matrices in spin space. The matrix's Green functions in Eq. (2) can be represented by the scaler Green's functions as

$$
\begin{gathered}
\hat{g}\left(\boldsymbol{r}, \theta, i \omega_{n}\right)=g\left(\boldsymbol{r}, \theta, i \omega_{n}\right) \hat{\sigma}_{0}, \\
\hat{f}\left(\boldsymbol{r}, \theta, i \omega_{n}\right)= \begin{cases}f\left(\boldsymbol{r}, \theta, i \omega_{n}\right)\left(-i \hat{\sigma}_{1}\right) & \text { for a triplet } \\
f\left(\boldsymbol{r}, \theta, i \omega_{n}\right) \hat{\sigma}_{2} & \text { for a singlet. }\end{cases}
\end{gathered}
$$

The pair potential in a chiral superconductor is described by

$$
\Delta(\boldsymbol{r}, \theta)=\Delta_{1}(\boldsymbol{r}) \cos (\chi \theta)+i \Delta_{2}(\boldsymbol{r}) \sin (\chi \theta),
$$

where $\theta$ is the azimuthal angle in the momentum space (i.e., $k_{x}=\cos \theta$ and $k_{y}=\sin \theta$ ), and $\Delta_{1}$ and $\Delta_{2}$ are the local amplitudes of two independent components. The topological numbers $\chi= \pm 1, \pm 2$, and \pm 3 characterize the chiral $p$-, chiral $d$-, and chiral $f$-wave superconductivities, respectively. The doubly degenerate chiral superconducting states are indicated by $\pm \chi$. In this study, we consider superconducting states with a positive $\chi$. Deep inside a superconductor (i.e., bulk region), the relation $\Delta_{1}=\Delta_{2}$ is satisfied. Therefore the pair potentials in the bulk are represented as

$$
\Delta(\theta)=\bar{\Delta}(T) e^{i \chi \theta},
$$

where $\bar{\Delta}(T)$ is the amplitude of the uniform pair potential at a temperature $T$. The amplitude of the superconducting gap is isotropic in momentum space. In the simulations, $\Delta_{1}$ and $\Delta_{2}$ are self-consistently determined by the gap equation,

$$
\left[\begin{array}{l}
\Delta_{1}(\boldsymbol{r}) \\
\Delta_{2}(\boldsymbol{r})
\end{array}\right]=N_{0} g_{0} \pi T \sum_{\omega_{n}} \int \frac{d \theta^{\prime}}{2 \pi} f\left(\boldsymbol{r}, \theta^{\prime}, i \omega_{n}\right)\left[\begin{array}{l}
V_{1}\left(\theta^{\prime}\right) \\
V_{2}\left(\theta^{\prime}\right)
\end{array}\right],
$$


where $N_{0}$ is the density of states per spin at the Fermi level in three dimensions. The coupling constant $g_{0}$ is determined by

$$
\left(N_{0} g_{0}\right)^{-1}=\ln \left(\frac{T}{T_{c}}\right)+\sum_{n=0}^{n_{c}} \frac{1}{n+1 / 2},
$$

where $n_{c}=\left(\omega_{c} / 2 \pi T\right)$ with $\omega_{c}$ being the cutoff energy. The functions $V_{1}$ and $V_{2}$ represent the attractive interactions as

$$
V_{1}(\theta)=2 \cos (\chi \theta), \quad V_{2}(\theta)=2 \sin (\chi \theta) .
$$

In our model, the Andreev bound states never appear at the surface in the $z$ direction (i.e., the top and bottom surfaces in Fig. 1) because the pair potential does not depend on $k_{z}$ [38]. As a result, the pair potential is less dependent on $z$. Thus, by setting the disk thin enough, $D \lesssim \xi_{0}<\lambda_{L}$, we ignore the $z$ dependence of the quasiclassical Green's functions. However, the thickness of the disk needs to be larger than the Fermi wavelength $1 / k_{F}$, so that the quasiclassical theory can be applied [40,41].

The effects of the rough surface are taken into account through the impurity self-energy, which is defined by

$$
\check{\Sigma}\left(\boldsymbol{r}, i \omega_{n}\right)=\left\{\begin{array}{cl}
\frac{i}{2 \tau_{0}} \int \frac{d \theta}{2 \pi} \check{g}\left(\boldsymbol{r}, \theta, i \omega_{n}\right) & \text { for } r>R-W, \\
0 & \text { for } r<R-W,
\end{array}\right.
$$

where $r=\left(x^{2}+y^{2}\right)^{1 / 2}$ and $\tau_{0}$ is the mean free time due to the impurity scatterings. The self-energy has finite values only near the surface, as shown in Fig. 1(b), where $W$ is the width of the disordered region.

The electric current $\boldsymbol{j}(\boldsymbol{r})$ is calculated from the Green's function

$$
\boldsymbol{j}(\boldsymbol{r})=\frac{\pi e v_{F} N_{0}}{2 i} T \sum_{\omega_{n}} \int \frac{d \theta}{2 \pi} \operatorname{Tr}\left[\check{T}_{3} \boldsymbol{k} \check{g}\left(\boldsymbol{r}, \theta, i \omega_{n}\right)\right],
$$

where $\breve{T}_{3}=\operatorname{diag}\left[\hat{\sigma}_{0},-\hat{\sigma}_{0}\right]$. The vector potential is determined by solving the Maxwell equation,

$$
\begin{gathered}
\nabla \times \boldsymbol{A}(\boldsymbol{r})=\boldsymbol{H}(\boldsymbol{r}), \\
\nabla \times \boldsymbol{H}(\boldsymbol{r})=4 \pi \boldsymbol{j}(\boldsymbol{r}) .
\end{gathered}
$$

In a finite-size superconductor, we define the amplitude of a spontaneous magnetization $M$ in terms of the spontaneous magnetic field $\boldsymbol{H}(\boldsymbol{r})$ as

$$
\boldsymbol{M}=\frac{1}{v} \int d \boldsymbol{r} \boldsymbol{H}(\boldsymbol{r})
$$

where $v=\pi R^{2} D$ is the volume of a small superconducting disk. In this paper, we did not calculate the magnetic field in three dimensions. We obtain $\boldsymbol{H}$ by solving the Maxwell equation in the $x-y$ plane with the boundary condition $H(x, y)=0$ outside of the disk, and assume that the magnetic field is homogeneous in the $z$ direction [i.e., $\boldsymbol{H}(\boldsymbol{r})=H(x, y) \hat{z}$ with $\hat{z}$ being the unit vector]. We iterate the Eilenberger equation for the Green's function and the Maxwell equation for the vector potential to obtain the self-consistent solutions of $\Delta_{1}(\boldsymbol{r}), \Delta_{2}(\boldsymbol{r}), \boldsymbol{A}(\boldsymbol{r})$, and $\check{\Sigma}\left(\boldsymbol{r}, i \omega_{n}\right)$.

We start all of the simulations with the initial conditions $\Delta_{1}(\boldsymbol{r})=\Delta_{2}(\boldsymbol{r})=|\bar{\Delta}(T)|$ and $\boldsymbol{A}(\boldsymbol{r})=0$, where $|\bar{\Delta}(T)|$ is the amplitude of the pair potential in a homogeneous superconductor at a temperature $T$. Throughout this paper, we fix several parameters: the radius of a disk $R=10 \xi_{0}$ and the cutoff energy $\omega_{c}=6 \pi T_{c}$. The magnetic field and the spontaneous magnetization are measured in units of the second critical magnetic field $H_{c_{2}}=\hbar c /|e| \xi_{0}^{2}$. The current density is normalized to $j_{0}=2|e| v_{F} N_{0} T_{c}=\hbar c^{2} / 4 \pi^{2}|e| \lambda_{L}^{2} \xi_{0}$. In the quasiclassical theory, the London length $\lambda_{L}=\left(m c^{2} / 4 \pi n_{e} e^{2}\right)^{1 / 2}$ with $n_{e}$ being the electron density is a parameter characterizing the spatial variation of magnetic fields and is fixed at $\lambda_{L}=5 \xi_{0}$. In this paragraph, we explicitly denoted $\hbar$ and $c$ to avoid misunderstandings.

To solve the Eilenberger equation in a disk geometry, we apply the Riccati parametrization to the Green's function [42-44] and the technique discussed in Ref. [45]. By using the Riccati parametrization, we can separate the Eilenberger equation into the two Riccati-type differential equations. Solving the Riccati equations along a sufficiently long quasiclassical trajectory (typically 30 times of the coherence length), we can obtain the solutions of the Eilenberger equation.

As we will demonstrate in the following sections, the edge currents show complicated spatial profiles depending on the pairing symmetry. To analyze such behaviors, we decompose the electric current into a series of current components in terms of the symmetry of Cooper pairs. By using the normalization relation $g^{2}-s_{v} f f=1$ under the assumption $f f \ll 1$, we represent the normal Green's function as $g \approx 1 \sim{ }^{\sim} s_{\nu} f f / 2$, where we have used the Eq. (6) and $s_{v}=1\left(s_{v}=-1\right)$ for the spin-triplet (spin-singlet) pair potential. By substituting the expression into the current formula in Eq. (13), the electric current can be expressed as [46]

$$
\begin{gathered}
\boldsymbol{j}(\boldsymbol{r})=\sum_{\omega_{n}>0} \boldsymbol{j}_{\omega_{n}}(\boldsymbol{r}), \\
\boldsymbol{j}_{\omega_{n}}=4 \pi e v_{F} N_{0} T \int \frac{d \theta}{2 \pi} \frac{1}{2} s_{v} \boldsymbol{k} \operatorname{Im}[f f]
\end{gathered}
$$

where we have used the relation $g\left(\boldsymbol{r}, \theta, i \omega_{n}\right)=$ $-g^{*}\left(\boldsymbol{r}, \theta,-i \omega_{n}\right)$. Generally speaking, the pairing function $f\left(\boldsymbol{r}, \theta, i \omega_{n}\right)$ can be decomposed into the Fourier series

$$
\begin{aligned}
f\left(\boldsymbol{r}, \theta, i \omega_{n}\right)= & \sum_{a=0} f_{a}^{\mathrm{c}}\left(\boldsymbol{r}, i \omega_{n}\right) \cos (a \theta) \\
& +\sum_{b=1} i f_{b}^{\mathrm{s}}\left(\boldsymbol{r}, i \omega_{n}\right) \sin (b \theta) .
\end{aligned}
$$

The surface breaks locally the inversion symmetry and induces subdominant pairing components whose symmetries are different from that of the pair potential. In the absence of the vector potential, $f_{a}^{\mathrm{c}}$ and $f_{b}^{\mathrm{s}}$ are real functions. When we consider the current profile at $y=0$, the electric current in the $y$ direction becomes

$$
\begin{gathered}
j_{y}(x)=\sum_{\omega_{n}} \sum_{a b} j_{a b}\left(i \omega_{n}\right), \\
j_{a b}\left(i \omega_{n}\right)=4 \pi|e| v_{F} N_{0} T f_{a}^{\mathrm{c}} f_{b}^{\mathrm{s}} I_{a b}, \\
I_{a b}=s_{v}(-1)^{b}\left(\delta_{b, 1-a}+\delta_{b, a+1}-\delta_{b, a-1}\right) / 4,
\end{gathered}
$$

where we use the relations $f\left(\boldsymbol{r}, \theta, i \omega_{n}\right)=f^{*}\left(\boldsymbol{r}, \theta+\pi, i \omega_{n}\right)$ and $\int d \theta \sin \theta \cos (a \theta) \sin (b \tilde{\theta})=\left(\delta_{b, 1-a}+\delta_{b, a+1}-\delta_{b, a-1}\right) \pi / 2$ for $a \geqslant 0$ and $b \geqslant 1$. The Kronecker's $\delta$ functions appearing 


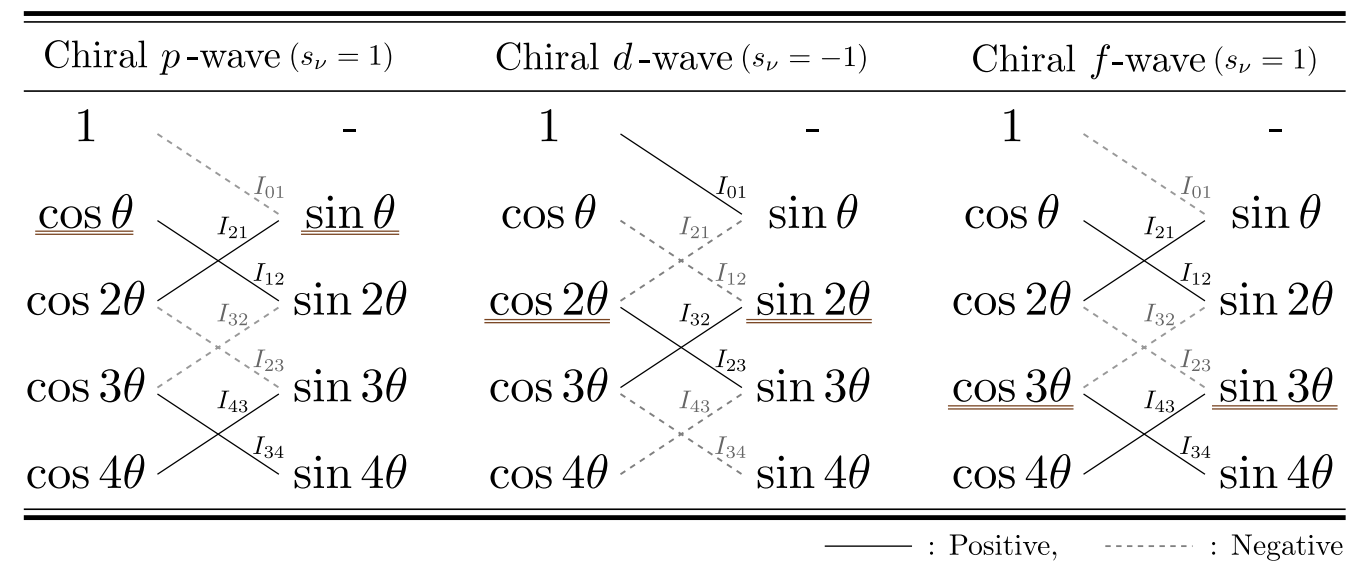

FIG. 2. Chart of $I_{a b}$ in Eq. (22). The diagonal lines connecting $\cos (a \theta)$ and $\sin (b \theta)$ indicate possible combinations of $f_{a}^{\mathrm{c}}$ and $f_{b}^{\mathrm{s}}$ for the chiral currents. The solid (broken) lines mean $I_{a b}$ is positive (negative). The sign of $I_{a b}$ in a spin-singlet superconductor is opposite to that in a spin-triplet superconductor due to an extra sign factor $s_{\nu}$. The double underlines indicate the principal pairing component linking to the pair potential [i.e., $\cos (\chi \theta)$ and $\sin (\chi \theta)$ ]. At the first line, "1" represents the $s$-wave component.

in Eq. (22) suggest that only the limited combinations of $f_{a}^{\mathrm{c}}$ and $f_{b}^{\mathrm{s}}$ contribute to the supercurrents, (e.g., $a=b \pm 1$ ). Moreover, the direction of the decomposed current $j_{a b}$ in Eq. (21) depends on the signs of $f_{a}^{\mathrm{c}} f_{b}^{\mathrm{s}}$ and $I_{a b}$. The signs of $I_{a b}$ mainly determine the current directions because $f_{a}^{\mathrm{c}} f_{b}^{\mathrm{s}}$ appearing at a certain surface have the same signs in most cases. We show a chart of $\operatorname{sgn}\left[I_{a b}\right]$ in Fig. 2. The diagonal lines connecting $\cos (a \theta)$ and $\sin (b \theta)$ mean the possible combinations for carrying the currents. The solid (broken) lines indicate that $I_{a b}$ is positive (negative). In a chiral $p$-wave superconductor, for example, $I_{01}$ and $I_{21}$ have the opposite signs of each other. As a result, the decomposed currents $j_{01}$ and $j_{21}$ flow in opposite directions.

\section{NON-SELF-CONSISTENT SIMULATION}

Before turning into the effects of surface roughness and those of the Meissner screening, the chiral currents in the uniform pair potential at $\boldsymbol{A}=0$ should be summarized. The results presented in this section are qualitatively the same as those obtained by the Bogoliubov-de Gennes (BdG) formalism in Refs. [17] and [18].

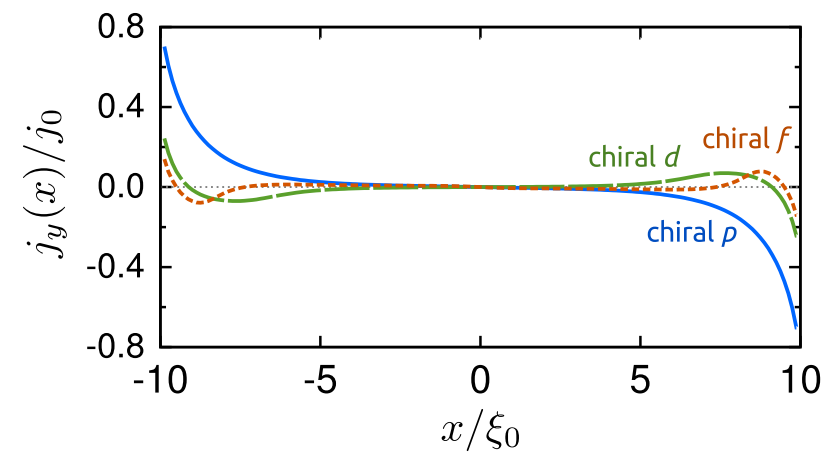

FIG. 3. Current densities in a disk of a chiral superconductor with a constant pair potential $\Delta_{1(2)}(\boldsymbol{r})=|\bar{\Delta}(T)|$ at $\boldsymbol{A}(\boldsymbol{r})=0$, where $|\bar{\Delta}(T)|$ is the amplitude of the pair potential at a temperature $T$ in a homogeneous superconductor. Here, we show the current distribution at $y=0$. The radius of a superconducting disk, the temperature, and the cutoff energy are set to $R=10 \xi_{0}, T=0.2 T_{c}$, and $\omega_{c}=6 \pi T_{c}$.
The spatial dependences of the edge current are shown in Fig. 3, where we show the spatial distribution of the current in the $y$ direction $j_{y}(x)$ at $y=0$, where the temperature is set to $T=0.2 T_{c}$. The results are circular symmetric on a superconducting disk. In a chiral $p$-wave superconductor $(\chi=1)$, the amplitude of the edge current takes its maximum at $r=R$ and monotonically decreases with increasing the distance from the surface. When we observe the current from the $+z$ axis, the chiral current flows in the clockwise direction. The current distributions in chiral $d$-wave $(\chi=2)$ and chiral $f$-wave $(\chi=3)$ superconductors are rather more complicated than that in a chiral $p$-wave case. The current density is negative (clockwise) around $x / \xi_{0}=10$ and is positive (counterclockwise) for $x / \xi_{0}<9$ in a chiral $d$-wave superconductor. In a chiral $f$-wave case, the current density is negative for $9.5<x / \xi_{0}<10$, positive for $7.8<x / \xi_{0}<9.5$, and negative again for $x / \xi_{0}<7.8$. The net current density $J=$ $\left.\int_{0}^{R} d x j_{y}(x)\right|_{y=0}$ decreases with increasing the chiral index $\chi$ because there are two (three) current channels in a chiral $d$-wave ( $f$-wave) superconductor and they carry the currents in opposite directions.

\section{DISK WITH A SPECULAR SURFACE}

In this section, we discuss the current distribution of a chiral-superconducting disk with a specular surface under the self-consistent pair potentials and the vector potential. The results are obtained by solving the Eilenberger and Maxwell equations simultaneously and self-consistently. In Sec. IV A, we consider only the self-consistent pair potential at $\boldsymbol{A}=0$ in Eq. (3) to analyze the complicated spatial distribution of the chiral current. The results tell us the symmetry of Cooper pairs that carry the chiral current. The effects of self-induced magnetic fields are briefly discussed in Sec. IV B. The parameters are set to the same values used in Fig. 3.

\section{A. Results under self-consistent pair potential at $\boldsymbol{A}=\mathbf{0}$}

In Fig. 4, we show the spatial dependence of the pair potentials $\Delta_{1}$ and $\Delta_{2}$. In a chiral $p$-wave superconductor, 


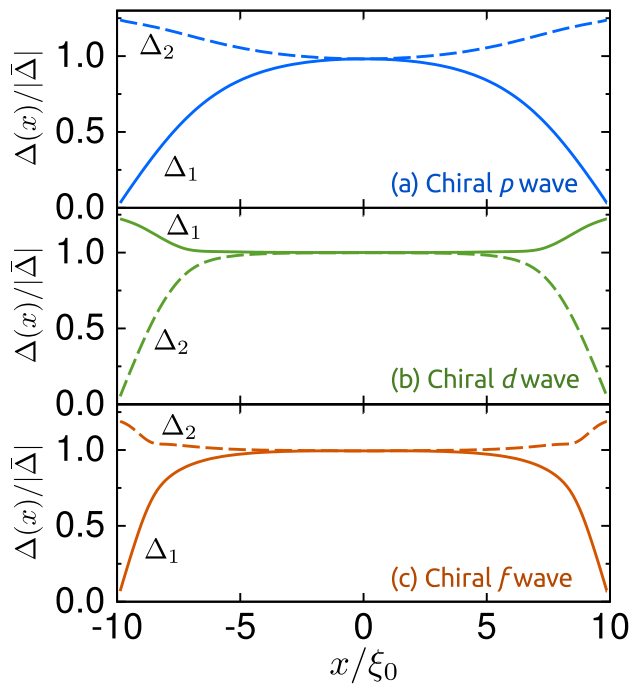

FIG. 4. Pair potentials in a disk of a chiral superconductor. The results are obtained by solving self-consistently the Eilenberger equation under the condition $\boldsymbol{A}(\boldsymbol{r})=0$. The superconducting disk is in the clean limit. The pair potentials are normalized to $|\bar{\Delta}(T)|$, the amplitude of the pair potential in a homogeneous superconductor at a temperature $T$. The parameters are set to the same values used in Fig. 3.

the pair potential $\Delta_{1}$ is strongly suppressed, whereas $\Delta_{2}$ is slightly enhanced near the surface as shown in Fig. 4(a). The suppression and enhancement are closely related to the formation of the surface ABSs. Namely, $\Delta_{1}$ changes its sign while the quasiparticle is reflected by a specular surface. These spatial variations of the pair potentials affect the edge current. The current density $j_{y}(x)$ at $y=0$ in Eq. (20) is shown in Fig. 5(a). In a chiral $p$-wave disk, the edge current monotonically decreases with increasing the distance from the edge. The edge current under the self-consistent pair potential in Fig. 5(a) flows in much wider area than that obtained by the uniform pair potential in Fig. 3. The range of the edge is determined by the spatial variation of the pair potential in Fig. 4.

The surface breaks locally the inversion symmetry and the spatial variation of the pair potential breaks the translational symmetry. As a result, the subdominant pairing correlations are induced near the surface [47]. In Fig. 2, we enumerate the orbital symmetry of such subdominant components. The double underlines indicate the principal pairing component linked to the pair potential. At the first row, 1 represents $s$-wave symmetry. In a chiral $p$-wave case, the spatial variation of the principal component $\cos (\theta)$ induces the subdominant component such as $s$-wave, $d$-wave $\cos (2 \theta), f$-wave $\cos (3 \theta)$, $\ldots$. In the same way, the principal component $\sin (\theta)$ induces the subdominant component of $d$-wave $\sin (2 \theta), f$-wave $\sin (3 \theta), \ldots$ The current is decomposed into the series of $j_{a b}$ in Eq. (21). The results for a chiral $p$-wave disk are shown in Fig. 5(b), where $j_{01}, j_{21}, j_{23}$, and $j_{43}$ contribute mainly to the current. Here $j_{a b}$ shown in Fig. 5(b) are calculated at the lowest Matsubara frequency $\omega_{0}$. We have confirmed that $\sum_{a b} j_{a b}\left(\omega_{0}\right)$ is almost identical to the current density obtained from the normal Green's function $j\left(\omega_{0}\right)$ and that

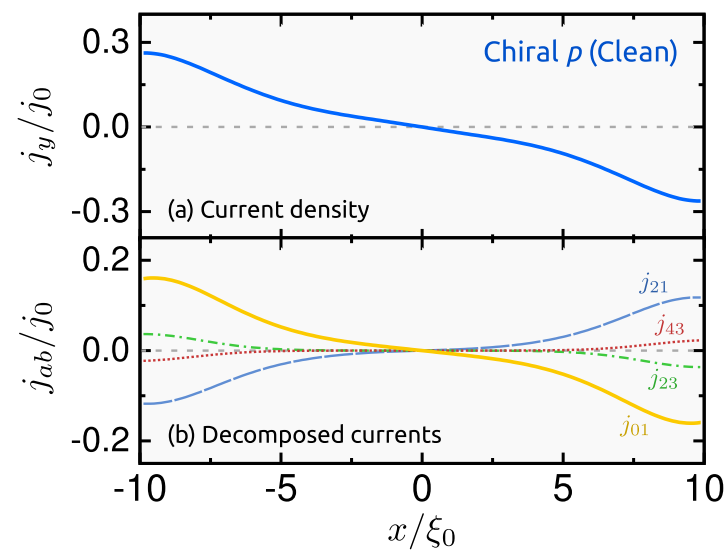

FIG. 5. Results for a chiral $p$-wave disk with a specular surface obtained by the self-consistent simulation under $\boldsymbol{A}(\boldsymbol{r})=0$. The chiral current $j_{y}(x)$ in Eq. (20) at $y=0$ is shown in panel (a). The decomposed current $j_{a b}$ at the lowest Matsubara frequency in Eq. (21) is shown in panel (b). All of the currents in panels (a) and (b) are normalized to $j_{0}=2|e| v_{F} N_{0} T_{c}$. The parameters are set to the same values used in Fig. 3.

the components at higher Matsubara frequencies have almost the similar spatial distribution as $j_{a b}$ at $\omega_{0}$. Reflecting the signs of $I_{a b}$ in Fig. 2, $j_{01}$ and $j_{23}$ flow in the clockwise direction, whereas $j_{21}$ and $j_{43}$ go in the counterclockwise direction. The magnitudes of $j_{01}$ and $j_{23}$ are slightly larger than $j_{21}$ and $j_{43}$, respectively. As a consequence, the net edge current flows in the clockwise direction. We have confirmed that other possible $j_{a b}$ are negligible. The decomposed currents in Fig. 5(b) tell us the symmetry of Cooper pairs that carry the edge current. The partial current $j_{01}$ represents the current carried by the combination of $s$-wave and $p_{y}$-wave Cooper pairs. The current $j_{21}$ are also understood as the current carried by $d_{x^{2}-y^{2}}$-wave $\times p_{y}$-wave Cooper pairs.

All of the Cooper pairs in a chiral $p$-wave superconductor belong to the spin-triplet symmetry class in the absence of spindependent potentials. Therefore, even-parity pairs induced at a surface have the odd-frequency symmetry because of the antisymmetry relation derived from the Fermi-Dirac statistics of electrons

$$
\hat{f}\left(\boldsymbol{r}, \theta, i \omega_{n}\right)=-\hat{f}^{\mathrm{T}}\left(\boldsymbol{r}, \theta+\pi,-i \omega_{n}\right),
$$

where $\cdot{ }^{\mathrm{T}}$ represents the transposition of a matrix and means the commutation of the two spins of a Cooper pair. The odd-parity symmetry accounts for the negative sign on the right-hand side of Eq. (23) in a spin-triplet superconductor. On the other hand, the induced spin-triplet even-parity components satisfy Eq. (23) by their frequency dependence. They are so-called odd-frequency Cooper pairs [48,49]. As shown in Fig. 5(b) and Eq. (21), the spontaneous edge current in a chiral superconductor is carried by the combination of the even- and odd-frequency Cooper pairs staying at a surface.

In a chiral $d$-wave superconductor, $\Delta_{2}$ is responsible for the formation of the surface ABSs. Correspondingly, the pair potential $\Delta_{1}$ is slightly enhanced near the surface as shown in Fig. 4(b). The spatial profile of the current is shown in Fig. 6(a). As is the case in the non-self-consistent simulation, there are two edge channels in a chiral $d$-wave disk. The current in the 


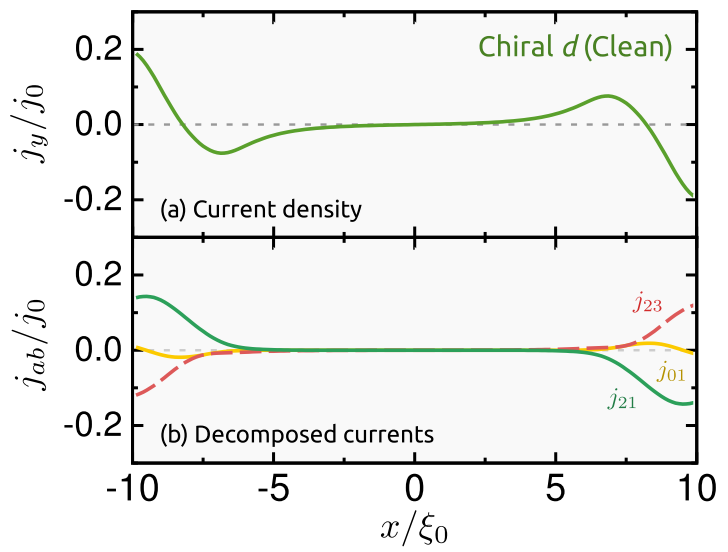

FIG. 6. Results for a chiral $d$-wave disk with a specular surface obtained by the self-consistent simulation under $\boldsymbol{A}(\boldsymbol{r})=0$ : current density $j_{y}$ (a) and dominant components $j_{a b}$ (b). The results are plotted in the same manner as in Fig. 5.

clockwise direction flows along the surface and the current in the counterclockwise flows around $x= \pm 7 \xi_{0}$. In Fig. 6(b), we decompose the current into the series of $j_{a b}$, where we show only dominant components of $j_{21}, j_{23}$, and $j_{01}$. We note that $j_{12}$ and $j_{32}$ (not shown) have almost the same profile as $j_{01}$ and that other components are negligible. The principal pairing components in a chiral $d$-wave superconductor are $f_{2}^{\mathrm{c}} \cos (2 \theta)$ and $f_{2}^{\mathrm{s}} \sin (2 \theta)$ as shown in Fig. 2. The spatial variation of the pair potential generates the odd-frequency components $f_{1}^{\mathrm{s}} \sin (\theta)$ and $f_{3}^{\mathrm{s}} \sin (3 \theta)$. These induced components carry the spontaneous current indicated by $j_{21}, j_{23}, j_{12}$, and $j_{32}$. As shown in Fig. 6(b), $j_{21}$ and $j_{23}$ flow in opposite directions because $I_{21}$ and $I_{23}$ have opposite signs. As a result, the net edge current becomes smaller than that in a chiral $p$-wave disk.

In a chiral $f$-wave disk, $\Delta_{1}$ is suppressed and $\Delta_{2}$ is slightly enhanced near the surface due to the emergence of the surface ABSs as shown in Fig. 4(c). The current profile and the decomposed currents $j_{a b}$ are shown in Figs. 7(a) and 7(b), respectively. Although the spatial profile of the current is greatly modified by the self-consistent pair potentials, Fig. 7(a) suggests that there are three current channels. The current density is negative for $8<x / \xi_{0}$, is positive for $6<x / \xi_{0}<8$, and is negative again for $0<x / \xi_{0}<6$. Figure 7(b) shows that the spatial dependence of the current components $j_{23}, j_{43}$, and $j_{34}$ are responsible for such a complicated current profile. We note that $j_{21}$ and $j_{32}$ (not shown) have almost the same profile as $j_{34}$.

\section{B. Results under self-consistent pair potential and vector potential}

We take into account the vector potential $\boldsymbol{A}$ induced by the edge current to investigate the Meissner screening effect. The pair potential and the vector potential are determined in a self-consistent way by solving the Eilenberger and Maxwell equations simultaneously. The spatial profiles of the pair potentials are qualitatively the same as those in Fig. 4. The spatial profiles of the chiral edge currents are shown in Fig. 8(a). In Figs. 8(b)-8(d), we compare the local magnetic fields obtained under the self-consistent field (SCF) with that under the non-self-consistent field of the vector

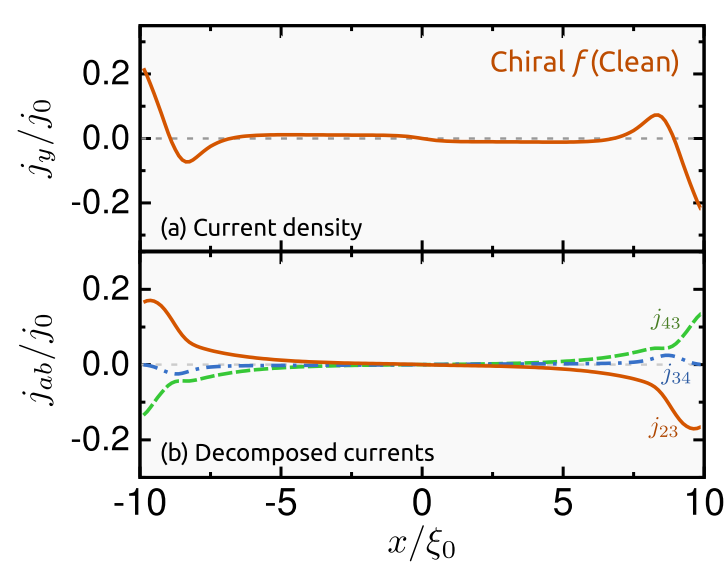

FIG. 7. Results for a chiral $f$-wave disk with a specular surface obtained by the self-consistent simulation under $\boldsymbol{A}(\boldsymbol{r})=0$ : current density $j_{y}$ (a) and dominant components $j_{a b}$ (b). The results are plotted in the same manner as Fig. 5.

potential (non-SCF). The latter is calculated from the current distribution in Figs. 5(a), 6(a), and 7(a) by using the relation in Eq. (15).

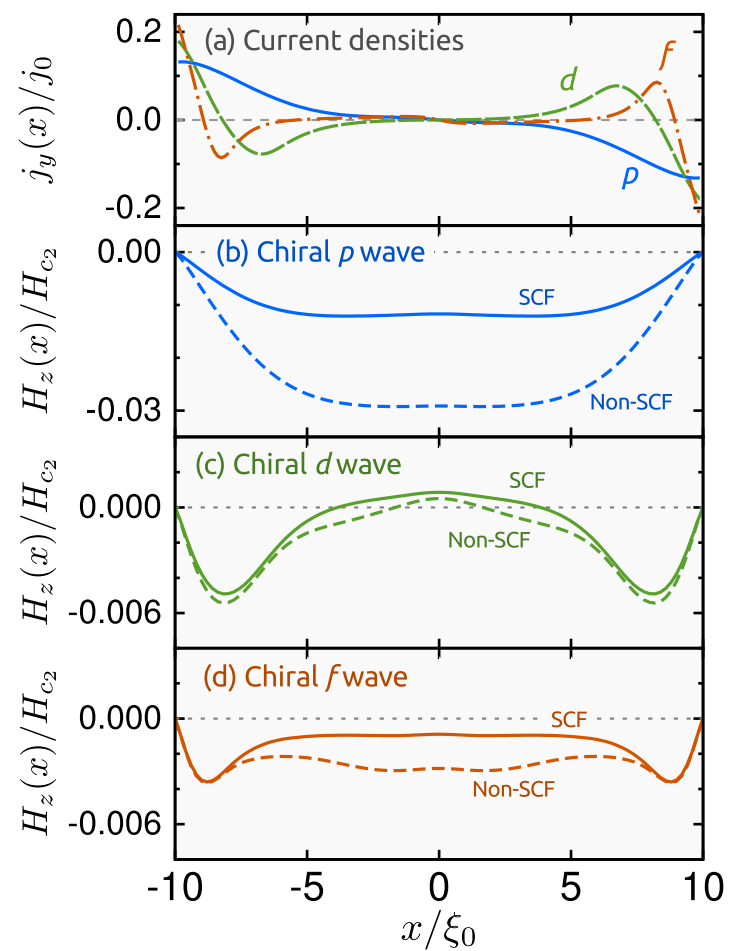

FIG. 8. (a) Current densities in a disk of a chiral superconductor with a specular surface (a). The results are obtained by solving the Eilenberger and Maxwell equations self-consistently and simultaneously. The penetration depth is fixed at $\lambda_{L}=5 \xi_{0}$. The other parameters are set to the same values used in Fig. 3. The current densities are normalized to $j_{0}=2|e| v_{F} N_{0} T_{c}$. In panels (b)-(d), we compare the spatial distributions of the self-consistent fields (SCF) with those of the non-self-consistent fields (non-SCF). The former is obtained by the current densities in panel (a) by using the relation in Eq. (15). The latter is calculated from the current distributions in Figs. 5(a), 6(a), and 7(a). The magnetic fields are scaled in units of $H_{c_{2}}=\hbar c /|e| \xi_{0}^{2}$. 


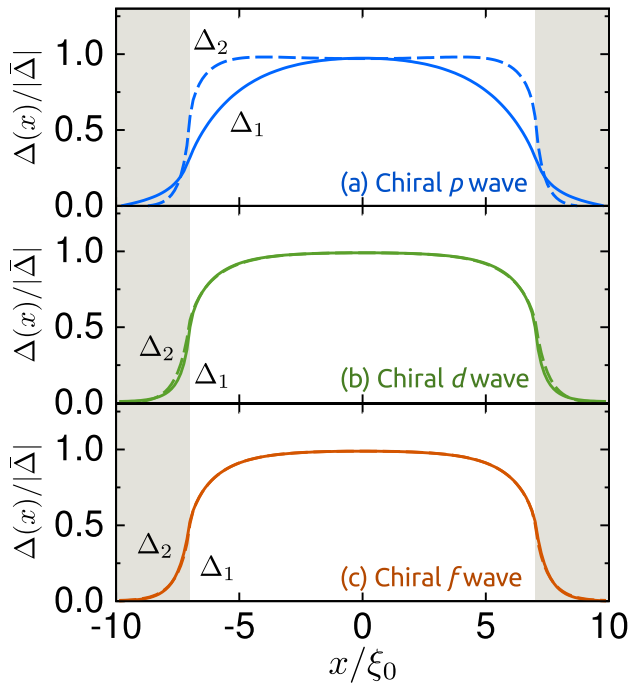

FIG. 9. Pair potentials in a disk of a chiral superconductor with a rough surface as indicated by the shadowed area. The results are obtained by solving the Eilenberger equation self-consistently at $\boldsymbol{A}=$ 0 . The parameters are set to the same values used in Fig. 3. The magnetic penetration depth is $\lambda_{L}=5 \xi_{0}$.

In a chiral $p$-wave disk, the Meissner screening by the superconducting condensate suppresses dramatically the spontaneous magnetization as shown in Fig. 8(b). As a result, the amplitude of the current at $x=R$ is less than $0.13 j_{0}$ under the SCF in Fig. 8(a), whereas it is about $0.27 j_{0}$ under the non-SCF in Fig. 5(a). The magnetic field near the center of a disk remains at a finite value in both the SCF and non-SCF simulations. This magnetic-field penetration is a results of the finite-size effects. At the surface of a semi-infinite sample, we have confirmed the current inversion because of the Meissner screening current as seen in Fig. 2 in Ref. [1]. Namely, the bulk condensate generates the screening current, which flows in the opposite direction to the chiral current at the surface. As a result, the magnetic field in the bulk region vanishes in a semi-infinite superconductor.

In a chiral $d$-wave superconductor, the magnetic field is mainly localized around $x= \pm 8 \xi_{0}$ as shown in Fig. 8(c). The results with the SCF are slightly smaller than those with the non-SCF. Thus the Meissner effect in a chiral $d$-wave disk is much weaker than that in a chiral $p$-wave one. The current profile under the non-SCF in Fig. 6(a) shows that there are two channels for the edge current. One is the outer channel for the clockwise current and the other is the inner channel for the counterclockwise current. The induced magnetic field by the inner current well screens that by the outer current intrinsically. Such a self-screening effect makes the Meissner screening effect weak in a chiral $d$-wave disk. Actually such characteristic current profiles with the non-SCF in Fig. 6(a) are well preserved in the results with the SCF in Fig. 8(a). The current amplitude at the surface $x=R$ reaches about $0.18 j_{0}$ in Fig. 8(a) and about $0.19 j_{0}$ in Fig. 6(a). Thus, in a chiral $d$-wave disk, the Meissner effect modifies the edge current only slightly, as shown in Fig. 8(c).

The result of the edge current for a chiral $f$-wave superconductor in Fig. 8(d) can be explained in the same way. There are three channels for the edge current in a chiral

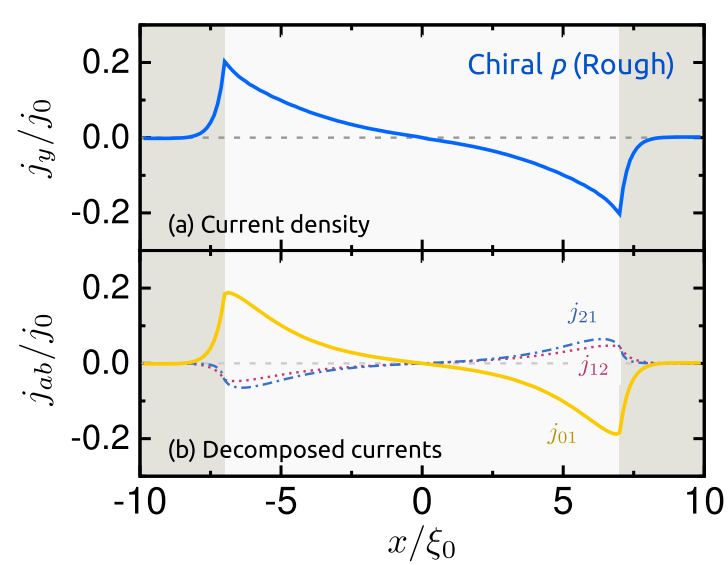

FIG. 10. Results for a chiral $p$-wave disk with a rough surface obtained by the self-consistent simulation at $\boldsymbol{A}(\boldsymbol{r})=0$. The current density $j_{y}(x)$ at $y=0$ in Eq. (20) is shown in panel (a). The dominant components $j_{a b}$ at the lowest Matsubara frequency are shown in panel (b).

$f$-wave case as discussed in Fig. 7(a). The self-screening effect works in this case as well. The characteristic behavior of the edge current with the non-SCF in Fig. 7(a) remains almost unchanged even with the SCF, as shown in Fig. 8(a). However, because the self-screening effect does not sufficiently exclude the local field, the magnetic field around the center of a disk is suppressed by the Meissner effect, as shown in Fig. 8(d).

\section{DISK WITH A ROUGH SURFACE}

In this section, we discuss the effects of a rough surface on the chiral edge currents. The width of the disordered region [shadowed in Fig. 1(b)] is set to be $W=3 \xi_{0}$ because the chiral edge current in the clean limit mainly flows in such area as shown in Fig. 8(a). The strength of roughness is set to $\xi_{0} / \ell=1.0$, where $\ell=v_{F} \tau_{0}$ is the elastic mean free path of a quasiparticle. The other parameters are set to the same values used in Fig. 5. The rough surface drastically changes the spatial profile of the pair potential and that of induced subdominant pairing components. Thus we first summarize symmetry of Cooper pairs appearing near the rough surface in Sec. V A. Then we discuss briefly the Meissner screening effect by the bulk condensate in Sec. V B.

\section{A. Results under self-consistent pair potential at $\boldsymbol{A}=\mathbf{0}$}

Here we discuss the results obtained by solving only the Eilenberger equation under the condition $\boldsymbol{A}=0$ in Eq. (3). We obtain the self-consistent solutions of $\Delta_{1}(\boldsymbol{r}), \Delta_{2}(\boldsymbol{r})$, and $\hat{\Sigma}\left(\boldsymbol{r}, i \omega_{n}\right)$. The pair potentials are presented in Fig. 9. The surface roughness strongly suppresses the pair potentials $\Delta_{1}$ and $\Delta_{2}$ in the disordered region of a chiral $p$-wave disk. At the interface between the disordered and clean regions (we refer to it as the d-c interface in what follows), $\Delta_{1}$ is suppressed more significantly than $\Delta_{2}$, which suggests the formation of the ABSs there [26]. We show the current density $j_{y}$ at $y=0$ and the dominant current components $j_{a b}$ in Fig. 10. Comparing Fig. 5(a) with 10(a), one can find that the peak of the edge current moves from the surface to the d-c interface, and that its maximum value $0.20 j_{0}$ is comparable to the maximum value 


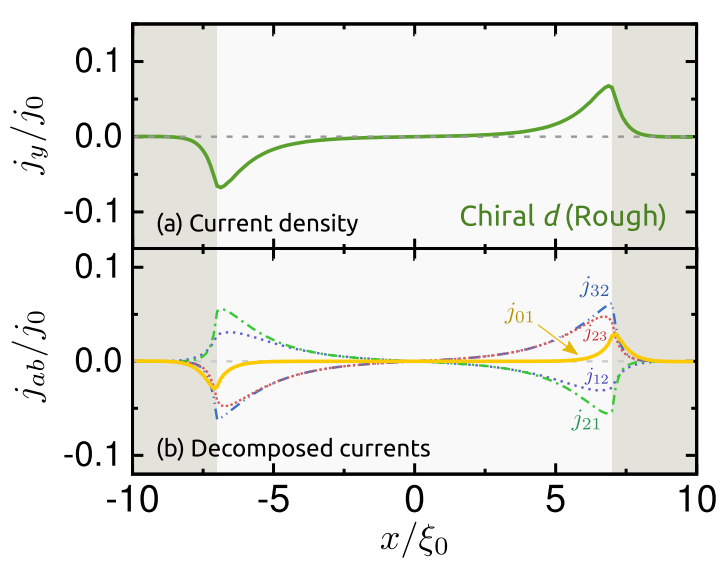

FIG. 11. Results for a chiral $d$-wave disk with a rough surface obtained by the self-consistent simulation at $\boldsymbol{A}(\boldsymbol{r})=0$ : current density $j_{y}$ (a) and dominant components $j_{a b}$ (b). The results are plotted in the same manner as Fig. 10.

in the clean limit. As shown in Fig. 10(b), the edge current in a chiral $p$-wave disk is mainly carried by three components; $j_{01}$, $j_{21}$, and $j_{12}$. Among them, the combination of $s$-wave $\times p_{y^{-}}$ wave pairs $\left(j_{01}\right)$ dominates obviously the chiral current in a disk with a rough surface. The spatial variation in $\Delta_{1}$ generates the $s$-wave and $d_{x^{2}-y^{2}}$-wave odd-frequency pairs [32]. The induced $s$-wave pairs, in particular, are robust even under the random potential. Such property supports the robustness of the chiral edge current in a chiral $p$-wave superconductor.

The edge current in a chiral $d$-wave disk shows a qualitatively different behavior from that in a chiral $p$-wave case. As shown in Fig. 11(a), the chiral current in a disk with surface roughness flows only in the counterclockwise direction. This behavior can be understood by comparing the current profile in Fig. 6(a) with that in Fig. 11(a). In the clean disk, there are two edge currents: the outer current running flowing in the clockwise direction and the inner current running in the counterclockwise direction as shown in Fig. 6(a). The surface roughness eliminates the outer current channel. However, the inner current channel remains even in the presence of the surface roughness and is responsible for the chiral current in the counterclockwise direction. We have confirmed that the inner current can survive in the presence of much stronger roughness such as $\xi_{0} / \ell \sim 30$. The decomposed components of the current $j_{a b}$ are shown in Fig. 11(b). The edge current is mainly carried by five combinations: $j_{01}, j_{12}, j_{21}, j_{23}$, and $j_{32}$. The four components $j_{12}, j_{21}, j_{23}$, and $j_{32}$ almost cancel one another. As shown in Fig. 9(b), the surface roughness suppresses both $\Delta_{1}$ and $\Delta_{2}$ in the same manner near the shadowed area, which results in

$$
f_{2}^{\mathrm{c}}(x) \simeq f_{2}^{\mathrm{s}}(x) .
$$

The spatial variation of $\Delta_{1}$ generates mainly $f_{1}^{\mathrm{c}} \cos (\theta)$ and $f_{3}^{\mathrm{c}} \cos (2 \theta)$ with $f_{1}^{\mathrm{c}}(x) \simeq f_{3}^{\mathrm{c}}(x)$. In the same way, the spatial variation of $\Delta_{2}$ induces $f_{1}^{\mathrm{s}} \sin (\theta)$ and $f_{3}^{\mathrm{s}} \sin (3 \theta)$ with $f_{1}^{\mathrm{s}}(x) \simeq$ $f_{3}^{\mathrm{s}}(x)$. Therefore, the relation

$$
f_{1}^{\mathrm{c}}(x) \simeq f_{3}^{\mathrm{c}}(x) \simeq f_{1}^{\mathrm{s}}(x) \simeq f_{3}^{\mathrm{s}}(x)
$$

holds among the four coefficients. By applying the relation in Eqs. (24) and (25) into Eq. (21) with the $I_{a b}$ in Fig. 2,

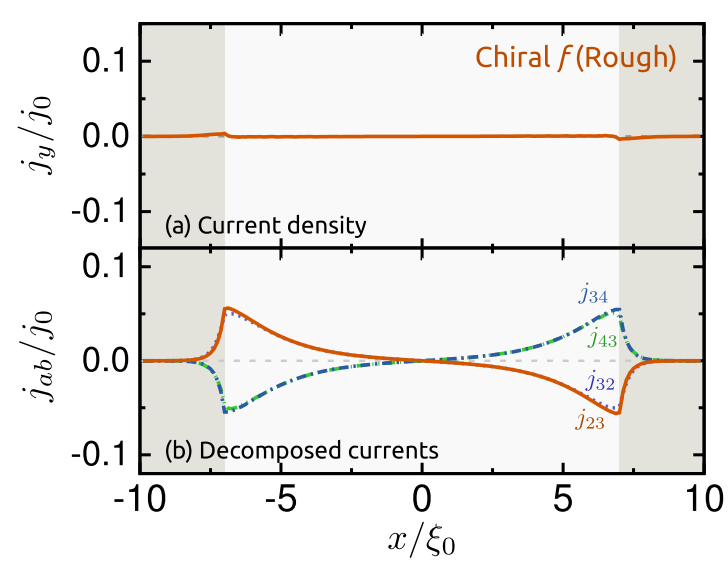

FIG. 12. Results for a chiral $f$-wave disk with a rough surface obtained by the self-consistent simulation at $\boldsymbol{A}(\boldsymbol{r})=0$ : current density $j_{y}$ (a) and dominant components $j_{a b}$ (b). The results are plotted in the same manner as in Fig. 10.

we can conclude that $j_{21}$ cancels $j_{32}$ and $j_{12}$ cancels $j_{23}$. The remaining component $j_{01}$, the contribution from the $s$-wave $\times p_{y}$-wave pairs, dominates the edge current. Because $s$-wave Cooper pairs are robust against surface roughness, $j_{01}$ can exist even under much stronger disordered potential.

As shown in Fig. 12(a), the edge current in a chiral $f$-wave disk with a rough surface becomes almost zero in this scale of the plot (i.e., $\left|j_{y}\right| \ll j_{0}$ ). Within the accuracy of our numerical simulation, the maximum value of the current density is less than $4 \times 10^{-3} j_{0}$. The dominant components $j_{23}, j_{32}, j_{34}$, and $j_{43}$ are shown in Fig. 12(b). As shown in Fig. 9(c), the surface roughness suppresses both $\Delta_{1}$ and $\Delta_{2}$ in the same manner near the shadowed area. By applying the same logic used in a chiral $d$-wave case, it is possible to show the relations

$$
\begin{gathered}
f_{3}^{\mathrm{c}}(x) \simeq f_{3}^{\mathrm{s}}(x), \\
f_{2}^{\mathrm{c}}(x) \simeq f_{4}^{\mathrm{c}}(x) \simeq f_{2}^{\mathrm{s}}(x) \simeq f_{4}^{\mathrm{s}}(x) .
\end{gathered}
$$

These relations and $I_{a b}$ in Fig. 2 explain the cancellation among the current components such as $j_{23}+j_{34} \simeq 0$ and $j_{32}+j_{43} \simeq$ 0 . As a result, the net edge current totally disappears, as shown in Fig. 12(a).

The symmetry of Cooper pairs is determined by the pair potential and the random impurity potential at a surface. Thus, even if a superconductor is semi-infinitely large and is realized with a single chiral domain, we can find the similar behavior of the edge currents against surface roughness as they show in a small superconductor.

\section{B. Results under self-consistent pair potential and vector potential}

We discuss the effects of the self-induced vector potential on the chiral current in a disk with surface roughness. By solving simultaneously the Eilenberger and Maxwell equations, we obtain the self-consistent solutions of $\Delta_{1}(\boldsymbol{r}), \Delta_{2}(\boldsymbol{r}), \boldsymbol{A}(\boldsymbol{r})$, and $\hat{\Sigma}\left(\boldsymbol{r}, i \omega_{n}\right)$. Here we do not show the pair potentials because they remain unchanged from those in Fig. 9 even quantitatively. The results of the edge currents are shown in Fig. 13(a). The spatial 


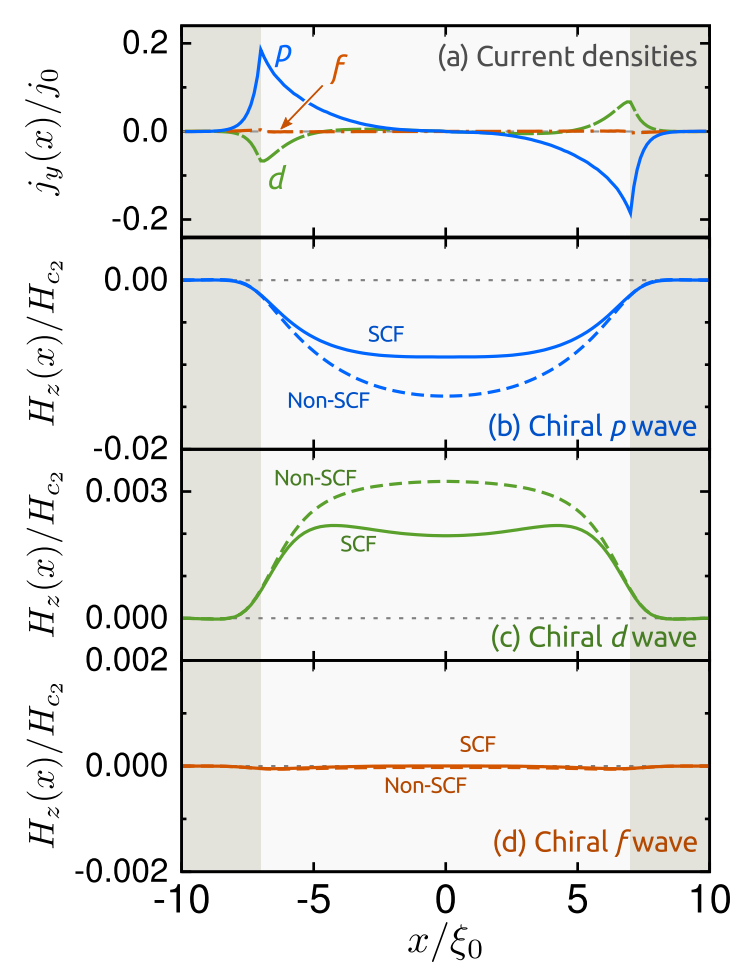

FIG. 13. (a) Current densities in a disk of a chiral superconductor with a rough surface. The results are obtained by solving the Eilenberger and Maxwell equations self-consistently and simultaneously. (b)-(d) Comparisons of the SCF with the non-SCF. The strength of the disorder and the width of the disordered region are set to $\xi_{0} / \ell=1$ and $W=3 \xi_{0}$, respectively. The other parameters are set to the same values used in Fig. 8.

distributions of the magnetic field are presented in Figs. 13(b)13(d). For comparison, we show the non-SCF calculated from the current profiles of Figs. 10(a), 11(a), and 12(a) by applying the relation in Eq. (15).

As shown in Fig. 13(b), the Meissner effect suppresses the magnetic field around the center of a chiral $p$-wave disk. When we compare the results for a chiral $p$-wave disk with the SCF in Fig. 13(a) and those with the non-SCF in Fig. 10(a), the current profile in Fig. 13(a) is spatially compressed into a narrower region by the Meissner effect.

Similar features are found also in the results of a chiral $d$-wave disk, as shown in Fig. 13(c). In the presence of the surface roughness, the current profile under the non-SCF has a monotonic spatial dependence between the center of the disk and the d-c interface as presented in Fig. 11(a). Therefore, the self-screening effect observed in a clean disk does not work at all. In a chiral $d$-wave disk with a rough surface, the Meissner effect becomes stronger than that in a disk with a specular surface. The Meissner effect suppress the magnetization near the center of the disk, as shown in Fig. 13(c).

In a chiral $f$-wave disk, the surface roughness strongly suppresses the chiral current. Thus the magnetic field is much smaller than $H_{c_{2}}$ everywhere in the disk, as shown in Fig. 13(d). Within the numerical accuracy, the magnetic field is less than $7 \times 10^{-5} H_{c_{2}}$ in our simulation.

\section{TEMPERATURE DEPENDENCE OF SPONTANEOUS MAGNETIZATIONS}

Finally, we discuss the dependences of the spontaneous magnetization on temperature, which are measurable values in experiments. All of the simulations were started at $T \simeq T_{c}$ with a homogeneous pair potential $\Delta_{1}(\boldsymbol{r})=\Delta_{2}(\boldsymbol{r})=|\bar{\Delta}(T)|$ and without an external magnetic field. The magnitude of a spontaneous magnetization is defined in Eq. (16). In our simulations, the pair potential, the impurity self-energy, and the vector potential are calculated self-consistently. The results in a disk with a specular surface are shown in Fig. 14. At a low temperature $T=0.1 T_{c}$, the magnetization of a chiral $p$-wave disk reaches to about $0.009 H_{c_{2}}$. In chiral $d$ - and $f$-wave disks, the magnetizations are about $0.002 H_{c_{2}}$. Although the magnetization decreases with increasing the radius of a disk by its definition, Eq. (16), $0.002 H_{c_{2}}$ at $R=10 \xi_{0}$ would be detectable value in experiments. The results in a disk with a rough surface are shown in Fig. 15; we choose $\xi_{0} / \ell=1.0$ and $W=3 \xi_{0}$. In a chiral $p$-wave superconductor, the amplitude of the magnetization is smaller than the results in the clean limit at every temperature. As we discussed in Sec. V, however, the amplitude of the current density in a disk with a rough surface is comparable to that in a disk with a specular surface. In Eq. (16), the magnetization is normalized by the area of a whole disk. As shown in Fig. 9, however, the effective radius of the superconducting region shrinks down to $R_{\text {eff }}=R-W$ in the presence of the surface roughness. When we renormalize the magnetization by the effective superconducting area, the renormalized magnetization $\tilde{M}=M R^{2} /(R-W)^{2} \approx 2 M$ is comparable to the magnetization in the clean disk. This fact means the robustness of the chiral current in the presence of the surface roughness.

In a chiral $d$-wave disk, the sign of the magnetization in Fig. 15 changes from that in Fig. 14 because only the inner chiral edge channel survives in a disk with a rough surface and flows the current in the counterclockwise direction. We have confirmed that the magnetization of a chiral $d$-wave disk becomes small but remains finite even in the presence of the much stronger disorder (e.g., $\xi_{0} / \ell=30$ ). As discussed in Sec. V A, the combination of $s$-wave and $p_{y}$-wave Cooper pairs carry the spontaneous current in both chiral $p$ - and chiral $d$-wave disks. Therefore, the robust spontaneous edge current and the robust spontaneous magnetization are common features in these two superconductors. In the case of a chiral $f$-wave superconductor, the amplitude of the magnetization is almost zero in the scale of Fig. 15. Within the numerical accuracy, we estimate that the magnetization is smaller than $4 \times 10^{-5} H_{c_{2}}$.

\section{CONCLUSION}

We have studied the effects of surface roughness on the spontaneous edge current in small chiral superconductors characterized by chiral $p$-, chiral $d$-, and chiral $f$-wave pairing symmetries. On the basis of the quasiclassical Eilenberger formalism, we calculated the chiral current and the spontaneous magnetization of the small superconducting disk numerically. By solving the Eilenberger and Maxwell equations simultaneously, we obtained self-consistent solutions of the 


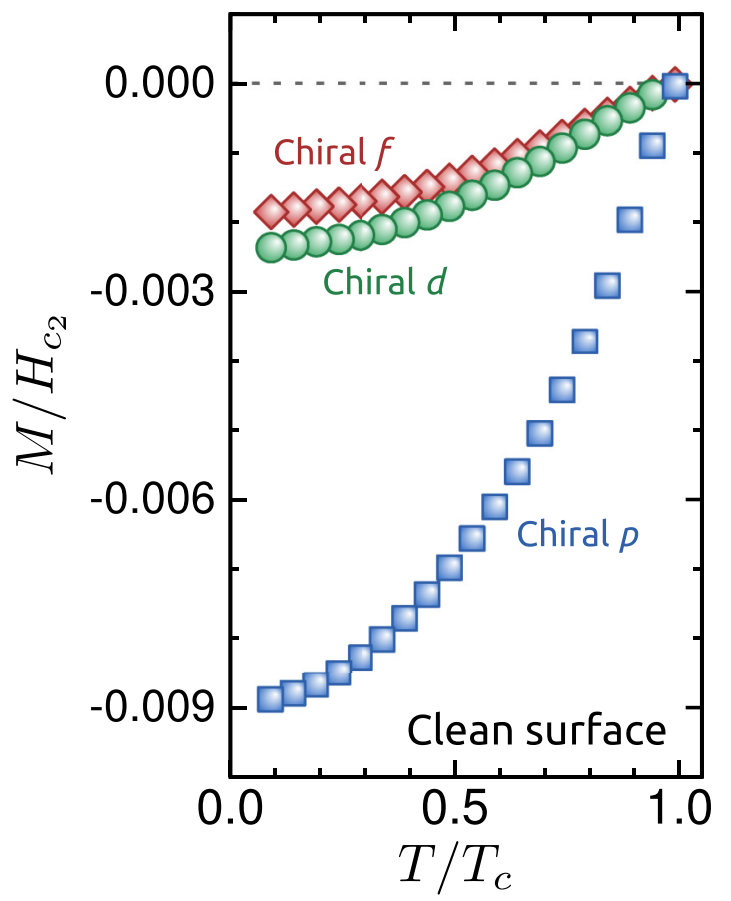

FIG. 14. Temperature dependences of the spontaneous magnetization of a small chiral superconductor with a clean surface. The magnetization is defined by Eq. (16) and is normalized to the second critical magnetic field $H_{c_{2}}$.

pair potential, the impurity self-energy, and the vector potential. To understand the physics behind the complicated current distribution in real space, we decomposed the current into a series of components in terms of the symmetry of a Cooper pair. The chiral edge current is carried by a combination of two pairing components: the even-parity component and the odd-parity component. In a spin-singlet (spin-triplet) superconductor, the odd-parity (even-parity) Cooper pairs have odd-frequency symmetry.

The effects of the surface roughness depend on the pairing symmetry of the superconductor. The chiral current is robust in the presence of surface roughness in a chiral $p$ - and chiral $d$-wave symmetries. With chiral $p$-wave symmetry, the characteristic features of the chiral current are insensitive to the surface roughness. With chiral $d$-wave symmetry, the chiral current changes its direction as a result of the surface roughness. In both the chiral $p$-wave and chiral $d$-wave cases, the chiral current is carried by a combination consisting of two pairing correlations. One is the correlation with $p$-wave symmetry and the other is the correlation with

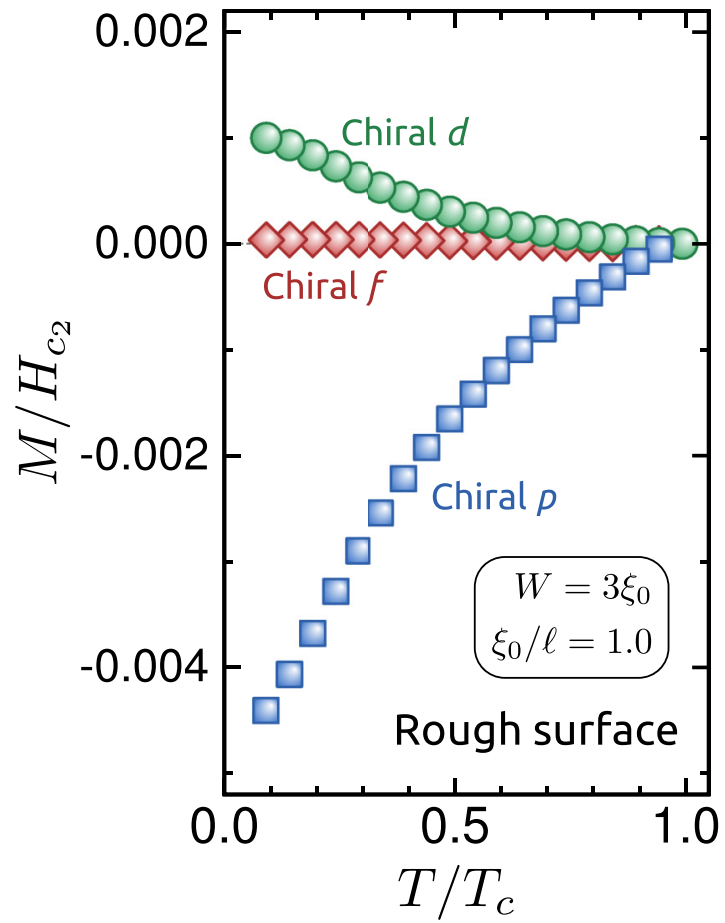

FIG. 15. Temperature dependences of the spontaneous magnetization of a small chiral superconductor with a rough surface. The parameters related to the surface roughness are set to $\xi_{0} / \ell=1.0$ and $W=3 \xi_{0}$.

$s$-wave symmetry. The Meissner screening effect by the bulk condensate reduces a spontaneous magnetization. However, the resulting amplitude of the magnetization is still large enough to be detected in experiments. In a chiral $f$-wave superconductor, the surface roughness significantly suppresses the spontaneous edge current.

\section{ACKNOWLEDGMENTS}

The authors are grateful to Y. Maeno, A. A. Golubov, Y. Tanaka, S. Kashiwaya, M. Ichioka, Ya. V. Fominov, and M. Yu. Kupriyanov for useful discussions. This work was supported by Topological Materials Science (No. 15H05852) and KAKENHI (No. 26287069 and No. 15H03525) from the Ministry of Education, Culture, Sports, Science, and Technology (MEXT) of Japan and by the Ministry of Education and Science of the Russian Federation (Grant No. 14Y.26.31.0007). S.-I.S. is supported in part by Grant-in-Aid for JSPS Fellows (Grant No. 15J00797) by the Japan Society for the Promotion of Science (JSPS).
[1] M. Matsumoto and M. Sigrist, J. Phys. Soc. Jpn. 68, 994 (1999).

[2] A. Furusaki, M. Matsumoto, and M. Sigrist, Phys. Rev. B 64, 054514 (2001).

[3] Y. Maeno, H. Hashimoto, K. Yoshida, S. Nishizaki, T. Fujita, J. G. Bednorz, and F. Lichtenberg, Nature (London) 372, 532 (1994).

[4] T. M. Rice and M. Sigrist, J. Phys. Condens. Matter 7, L643 (1995).
[5] A. P. Mackenzie and Y. Maeno, Rev. Mod. Phys. 75, 657 (2003).

[6] K. Takeda, H. Sakurai, E. Takayama-Muromachi, F. Izumi, R.

A. Dilanian, and T. Sakai, Nature (London) 422, 53 (2003).

[7] G. Baskaran, Phys. Rev. Lett. 91, 097003 (2003).

[8] M. Ogata, J. Phys. Soc. Jpn. 72, 1839 (2003).

[9] A. Tanaka and X. Hu, Phys. Rev. Lett. 91, 257006 (2003).

[10] R. Nandkishore, L. S. Levitov, and A. V. Chubukov, Nat. Phys. 8, 158 (2012). 
[11] M. L. Kiesel, C. Platt, W. Hanke, D. A. Abanin, and R. Thomale, Phys. Rev. B 86, 020507(R) (2012).

[12] M. L. Kiesel, C. Platt, W. Hanke, and R. Thomale, Phys. Rev. Lett. 111, 097001 (2013).

[13] M. H. Fischer, T. Neupert, C. Platt, A. P. Schnyder, W. Hanke, J. Goryo, R. Thomale, and M. Sigrist, Phys. Rev. B 89, 020509(R) (2014).

[14] Y. Kasahara, H. Shishido, T. Shibauchi, Y. Haga, T. D. Matsuda, Y. Onuki, and Y. Matsuda, New J. Phys. 11, 055061 (2009).

[15] H. Kusunose, J. Phys. Soc. Jpn. 81, 023704 (2012).

[16] M. Tsuchiizu, Y. Yamakawa, S. Onari, Y. Ohno, and H. Kontani, Phys. Rev. B 91, 155103 (2015).

[17] Y. Tada, W. Nie, and M. Oshikawa, Phys. Rev. Lett. 114, 195301 (2015).

[18] W. Huang, E. Taylor, and C. Kallin, Phys. Rev. B 90, 224519 (2014).

[19] P. G. Björnsson, Y. Maeno, M. E. Huber, and K. A. Moler, Phys. Rev. B 72, 012504 (2005).

[20] J. R. Kirtley, C. Kallin, C. W. Hicks, E.-A. Kim, Y. Liu, K. A. Moler, Y. Maeno, and K. D. Nelson, Phys. Rev. B 76, 014526 (2007).

[21] J. A. Sauls, Phys. Rev. B 84, 214509 (2011).

[22] K. Nagai, J. Low Temp. Phys. 175, 44 (2014).

[23] K. Saitoh, S. Kashiwaya, H. Kashiwaya, M. Koyanagi, Y. Mawatari, Y. Tanaka, and Y. Maeno, Appl. Phys. Express 5, 113101 (2012).

[24] K. Saitoh, S. Kashiwaya, H. Kashiwaya, Y. Mawatari, Y. Asano, Y. Tanaka, and Y. Maeno, Phys. Rev. B 92, 100504(R) (2015).

[25] P. E. C. Ashby and C. Kallin, Phys. Rev. B 79, 224509 (2009).

[26] S. V. Bakurskiy, A. A. Golubov, M. Yu. Kupriyanov, K. Yada, and Y. Tanaka, Phys. Rev. B 90, 064513 (2014).

[27] S. Lederer, W. Huang, E. Taylor, S. Raghu, and C. Kallin, Phys. Rev. B 90, 134521 (2014).

[28] A. Bouhon and M. Sigrist, Phys. Rev. B 90, 220511(R) (2014).

[29] W. Huang, S. Lederer, E. Taylor, and C. Kallin, Phys. Rev. B 91, 094507 (2015).

[30] T. Scaffidi and S. H. Simon, Phys. Rev. Lett. 115, 087003 (2015).

[31] S.-I. Suzuki and Y. Asano, Phys. Rev. B 89, 184508 (2014).
[32] S.-I. Suzuki and Y. Asano, Phys. Rev. B 91, 214510 (2015).

[33] L. J. Buchholtz and G. Zwicknagl, Phys. Rev. B 23, 5788 (1981).

[34] J. Hara and K. Nagai, Prog. Theor. Phys. 76, 1237 (1986).

[35] S. Kashiwaya, Y. Tanaka, N. Terada, M. Koyanagi, S. Ueno, L. Alff, H. Takashima, Y. Tanuma, and K. Kajimura, J. Phys. Chem. Solids 59, 2034 (1998).

[36] C. R. Hu, Phys. Rev. Lett. 72, 1526 (1994).

[37] Y. Tanaka and S. Kashiwaya, Phys. Rev. Lett. 74, 3451 (1995).

[38] Y. Asano, Y. Tanaka, and S. Kashiwaya, Phys. Rev. B 69, 134501 (2004).

[39] G. Eilenberger, Z. Phys. 214, 195 (1968).

[40] A. F. Andreev, Zh. Eksp. Teor. Fiz. 46, 1823 (1964) [Sov. Phys. JETP 19, 1228 (1964)].

[41] Y. Nagato and K. Nagai, Phys. Rev. B 51, 16254 (1995).

[42] N. Schopohl and K. Maki, Phys. Rev. B 52, 490 (1995).

[43] N. Schopohl, arXiv:cond-mat/9804064 (unpublished).

[44] M. Eschrig, Phys. Rev. B 80, 134511 (2009).

[45] Y. Nagai, K. Tanaka, and N. Hayashi, Phys. Rev. B 86, 094526 (2012).

[46] S. Higashitani, J. Phys. Soc. Jpn. 83, 075002 (2014).

[47] In this paper, we assume only the principal pairings (e.g., $p_{x}$ - and $p_{y}$-wave pairs in a chiral $p$-wave superconductor) are attractive. Nevertheless, subdominant pairing correlations belonging to different symmetry classes from that of the dominant one appear near a surface. The pairing correlations $f$ and the order parameters $\Delta$ are directly connected by the gap equation Eq. (9). Subdominant order parameters never appear in our formulation because we set the attractive interactions to be zero for subdominant pairing correlations. These subdominant pairing correlations induced by a surface are not related to the stability of a superconductor because they cannot become order parameters.

[48] V. L. Berezinskii, Pis'mav Zh. Eksp. Teor. Fiz. 20, 628 (1974) [Sov. Phys. JETP Lett. 20, 287 (1974)].

[49] Y. Tanaka, M. Sato, and N. Nagaosa, J. Phys. Soc. Jpn. 81, 011013 (2012). 